\title{
Radial Distributions of Dark and Luminous Matter in Bright Spiral Galaxies
}

\author{
Susan A. Kassin \\ Department of Astronomy, The Ohio State University, 140 W. 18th \\ Ave., Columbus, OH 43210-1173, USA. \\ Roelof S. de Jong \\ Space Telescope Science Institute, 3700 San Martin Drive, Baltimore, \\ MD 21218, USA.
Richard W. Pogge
Department of Astronomy, The Ohio State University, $140 \mathrm{~W} .18$ th Ave., Columbus, OH 43210-1173, USA.

\begin{abstract}
We present a work in progress investigating the radial distributions of dark and luminous matter in local bright spiral galaxies spanning a range of Hubble types. The distributions are derived from multiband imaging (BVRJHK) predominately from the Ohio State University Bright Spiral Galaxy Survey (OSUBSGS) and rotation curves from the literature. We use stellar colour-M/L relationships from Bell \& de Jong (2001, ApJ, 550, 212) to create radial stellar mass surface density profiles for each of our galaxies. These profiles are used to create rotation curves for the stellar component; gas masses are derived from $\mathrm{HI}$ when available. The resulting stellar and gas mass rotation curves are compared to observed (total mass) rotation curves, and the rotation due to dark matter is determined for each galaxy. Fits to Navarro, Frenk, \& White (1996, ApJ, 462, 563; NFW) halo profiles are shown. We present this analysis for 2 example galaxies from our sample of nearly 50 . We will use this data to investigate correlations between these mass components and physical properties of galaxies.
\end{abstract}

In Fig. 1 \& 2 we show two examples of galaxies for which we have created radial luminous and dark matter profiles. For each galaxy, dark matter profiles predicted by NFW are adiabatically contracted according to the baryonic mass distribution of the galaxy. A rotation curve for the resulting contracted NFW profile is then fit to the observed rotation curve of the galaxy. $\chi^{2}$ contours are plotted for fits to the concentration parameter, $c$, and the velocity at $r_{200}$, $\mathrm{v}_{200}$. (The radius at which the mean density of the halo is 200 times the critical density is defined as $r_{200}$.) 

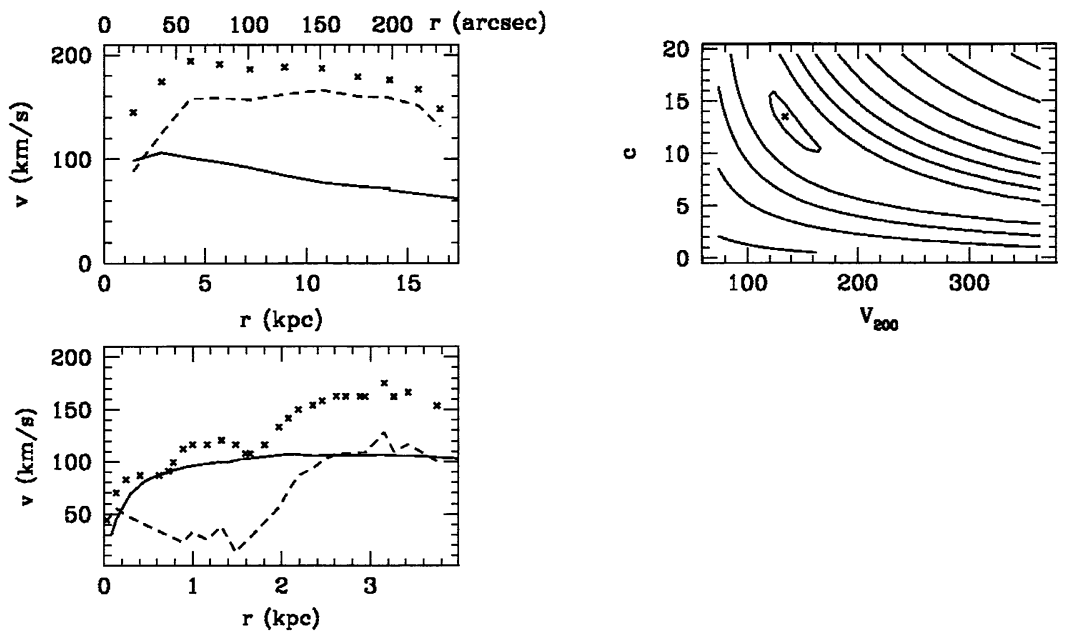

Figure 1. Dark and luminous mass rotation curves for NGC 3893, an $\mathrm{SAB}(\mathrm{rs}) \mathrm{c}$ : galaxy, are plotted at left. Total mass rotation curves are plotted as X's, luminous mass as a solid line, and dark mass as a dashed line. The upper left panel is for an HI rotation curve (Verheijen, M. A. W. $1997 \mathrm{PhD}$ thesis Univ. Groningen, The Netherlands), and the bottom left panel is for an $\mathrm{H} \alpha$ rotation curve (Kranz, T. 2002, $\mathrm{PhD}$ thesis Universitat Heidelberg, Germany). Dark matter begins to dominate the galaxy's rotation at $\sim 2.5 \mathrm{kpc}$. A fit to an NFW profile is shown at right; contours are 0.2 in $\chi^{2}$. NGC 3893 is among our best constrained galaxies, even though it shows a banana-shaped $\chi^{2}$ space, and the $1 \sigma$ contour is quite large. Its best-fit parameters are $\mathbf{c}=13.5$ and $\mathrm{v}_{200}=134 \mathrm{~km} / \mathrm{s}$.
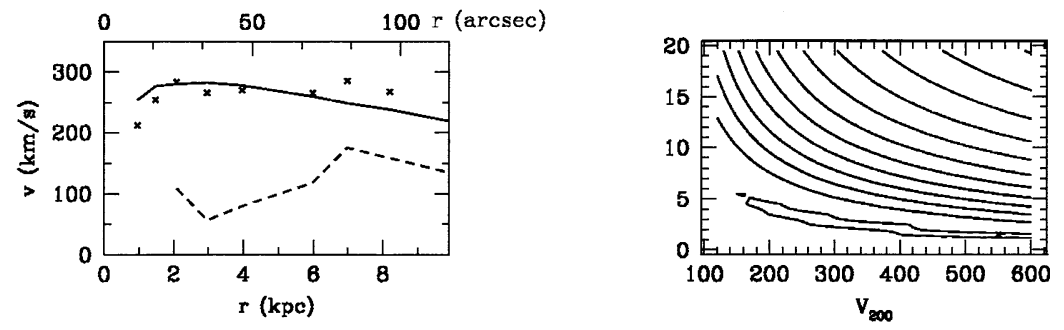

Figure 2. Same as Figure 1, except for NGC 7217, an (R)Sa(r)ab galaxy. The $\mathrm{H} \alpha$ rotation curve is from Kent $(1986, \mathrm{AJ}, 91,1301)$. This galaxy shows a decrease in the role of stellar mass at $\sim 6 \mathrm{kpc}$. NGC 7217 does not have a constrained fit to an NFW profile. 